\title{
FIBROMA OF METACARPAL- RARE PRESENTATION.
}

Varunjikar, ${ }^{1}$, Mrs. Varunjikar A. M ${ }^{2}$, Mrs. S. C. Joshi³, C. R. Joshi

1. Associate Professor, Department of Orthopedics, Vikhe Patil Hospital \& Medical College, Ahmednagar, Maharashtra.

2. Consultant anaesthesiologist, Department of Anaesthesiology, Aryangla Medical College, Satara.

3. Consultant Anaesthesiologist, SR-M G M, Hospital, Parel, Mumbai.

4. Consultant Radiologist, Dept of Radiology - M G M Hospital, Parel, Mumbai.

\section{CORRESPONDING AUTHOR:}

Dr. Varunjikar,

Tathastu 54 Deshmukh Colony, Camp, Satara- 415001.

E-mail: varunhsp@yahoo.co.in

\section{HOW TO CITE THIS ARTICLE:}

Varunjikar, Mrs. Varunjikar A M, Mrs. S. C Joshi³, C. R. Joshi. "Fibroma of Metacarpal- Rare Presentation". Journal of Evolution of Medical and Dental Sciences 2013; Vol2, Issue 26, July 1; Page: 4659-4662.

ABSTRACT: Benign and malignant affections of bone is not uncommon entity. Both types of lesions are seen in both sexes and all age groups. Ossifying fibroma is a rare, benign, slow-growing bone tumor. Tumor has a high recurrence rate. This report describes an ossifying fibroma of the right second metacarpal in a 25-year-old female. Our aim is to show successful treatment of a metacarpal ossifying fibroma with wide resection.

KEY WORDS: Ossifying fibroma, metacarpal, radiograph and intra operative photographs.

INTRODUCTION: Metacarpal fibroma is a rare, benign, slow-growing bone tumor. The tumor was first described in 1948 by Jaffe and Lichtenstein who differentiated the histologic findings from that of chondrosarcoma and enchondroma ${ }^{2}$, 3. In its etiology, chromosome anomaly and immunologic factors have been proposed 4 . The tumor is rare and accounts for less than $1 \%$ of primary bone tumours ${ }^{1}$. In the period from 1948 to 2012, more than 700 cases have been reported in the literature $^{2}$. It is generally seen in the metaphysis of lower extremity long bones, most frequently involving proximal tibial metaphysis (80\%) ${ }^{1}$. It is extremely uncommon in the bones of the hand and less than 30 cases fibroma affecting the hand and metacarpal bones have been reported ${ }^{2}$. It is more frequent in men than in women ${ }^{5}$, primarily affecting young adults in their second and third decades of life, $80 \%$ of patients are younger than 36 years. Histologic findings may show lesion consisted of immature-looking cartilage with myxoid and fibrous components ${ }^{7}$. Fibroma is usually slow-growing, sharply demarcated tumor, sometimes it may behave in an aggressive way destroying trabecular bone and extending into soft tissues, malignant conversion is extremely rare. Tumor has a high recurrence rate, up to $25 \%{ }^{8}$. Most of the patients are asymptomatic for a long period; pain is the most common symptom and may become more severe with time ${ }^{9}$. Patients may also report local swelling, palpable mass and in very rare cases, a limitation of joint motion ${ }^{1,2}$. 
In the differential diagnosis chondromyxoid fibroma, chondrosarcoma, chondroblastoma, enchondroma, nonossifying fibroma and aneurysmal bone cyst should be included 10 . We report a case of metacarpal fibroma involving right second metacarpal bone of the 25 year old women.

CASE REPORT: 25yrs old female, came with c/o swelling over dorsum of right hand since 1 year, gradually progressive with pain which increased on movement of fingers \& relieved on rest

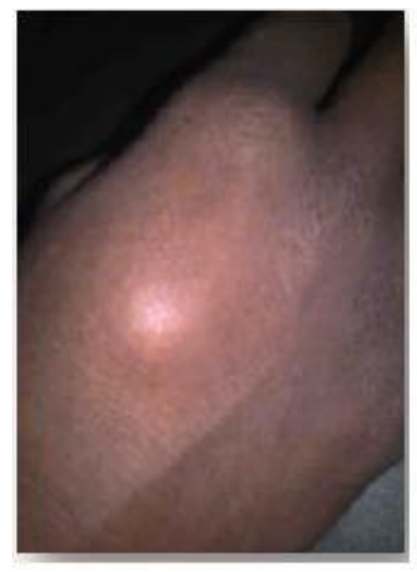

Pre-operative clinical photograph of dorsum of hand showing swelling arising from second metacarpal

Local examination revealed, swelling approx $1 \times 2 \mathrm{~cm}$ in dorsum of hand in the region of $2^{\text {nd }}$ metacarpal diaphyseal region, non-mobile, firm in consistency, diffuse margins, non-fluctuant, fixed to underlying bone. Provisional diagnosis of bone cyst was made, lab investigations revealed- $\mathrm{Hb}$ 10.5 gm\%, ESR 15 at end of $1^{\text {st }}$ hour, urine microscopic shows amorphous crystals, BSL fasting 90 mg, PP $140 \mathrm{mg} \%$, RFT and LFT were within normal limits.

$\mathrm{X}$-ray shows sclerosis and periosteal reaction.

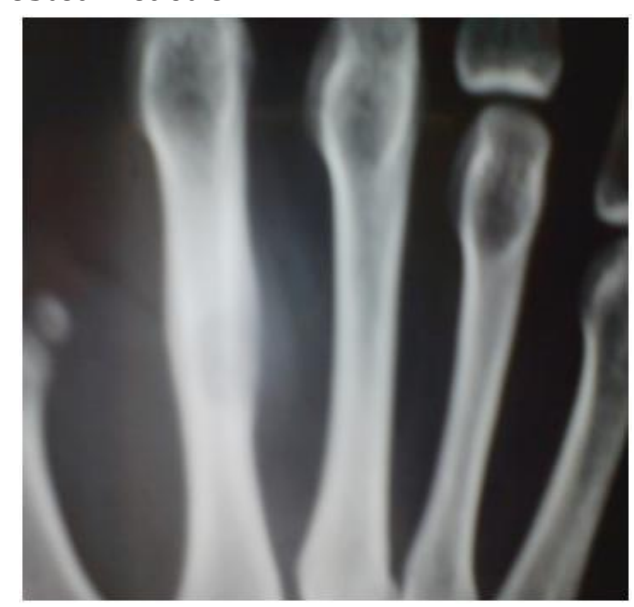

X-ray showing sclerosis of the shaft of second metacarpal and timorous shadow seen extending out of the shaft towards $3^{\text {rd }}$ metacarpal.

After obtaining informed consent, patient underwent excision of swelling under anaesthesia \& sample was sent for histopathology. 
Post- operative, cock-up slab was given which was removed after 3 wks, stitches were removed after 10 days, and recovery was uneventful. Patient was advised active finger exercises after 3 weeks.

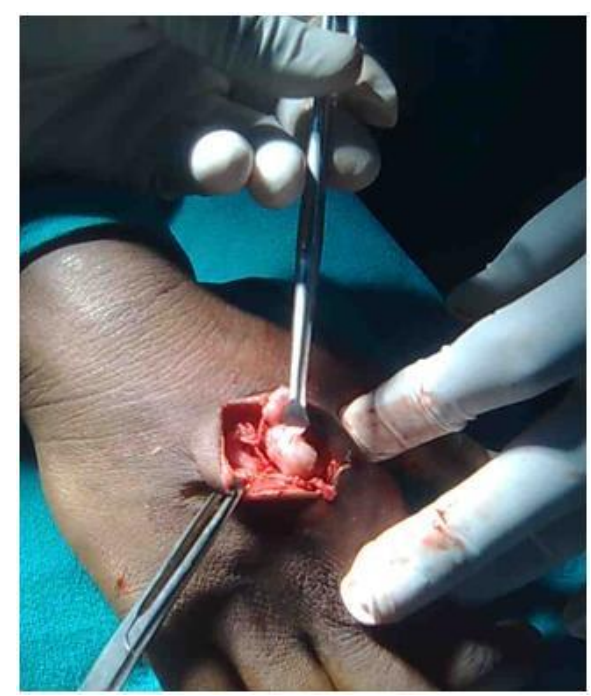

Intraoperative photograph of excision of swelling arising from second metacarpal.

Biopsy revealed nests, cords of round to oval cells with indistinct cytoplasm with fibrosis and osteoid formation; lobulated; surrounded by partial capsule of mature bone; with minimal atypia and minimal mitotic figures, small areas of necrosis, vascular invasion, and high nuclear grade were seen, thus a diagnosis of ossifying fibroma of metacarpal was made.

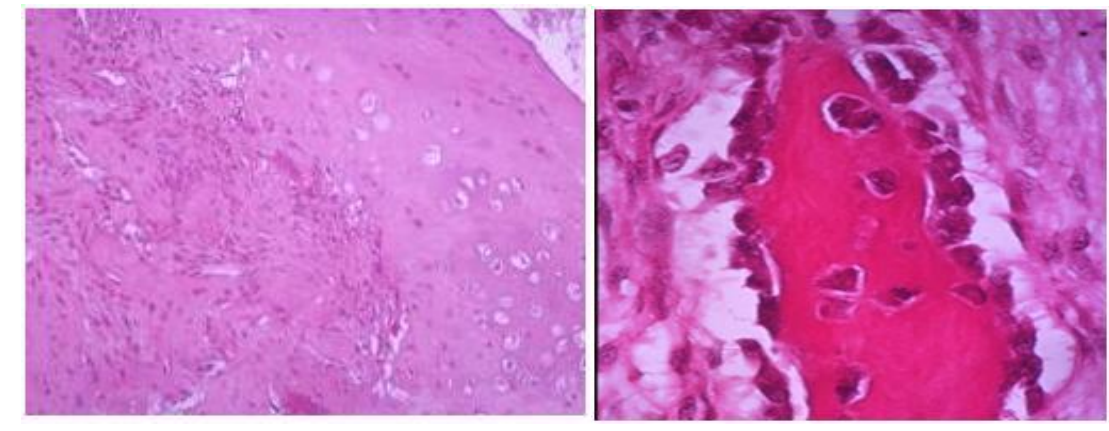

Slides showing histo pathological features of ossifying fibroma as stated in the script.

Patient was followed up and assessed clinically and radiologically at period of about 3 months, 6 months and 1 year. Patient has recovered fully and performing day to day activities.

DISCUSSION: The metacarpal fibroma described here arose in 25 year old female patient which correlate with literature reports about the age of patients. It is generally seen in patients at age of 30. It has been reported that it is seen somewhat more in men than in women. The lesion generally occurs in the metaphysis of long bones and most commonly involved bone is tibia. It is rare in the bones of the hand. In our case tumor is affecting diaphyseal region of metacarpal bone and as far as we know only few cases had been reported until now. The patients generally present with a complaint of pain and local swelling, in some cases there may be problems related to movement of 
the joints. Our patient had similar symptoms. On plain radiographs cortical sclerosis and expansile lesion are generally seen6. Roentgenographic picture in our case was sclerotic in nature. In some cases, magnetic resonance imaging and CT may demonstrate spread to soft tissue, with our patient that wasn't a case. Positive stains for tumor: S100 (60\%), vimentin, Leu7/CD57 (focal), GFAP (focal), desmin (13\%), pan-keratin (10\%).Tumor has a high recurrence rate, up to $25 \% 9$. Some studies reported recurrence rate from $7 \%$ to $80 \%$, depending on the treatment ${ }^{11}$. Lersundi et al. found a $50 \%$ recurrence rate in patients treated with curettage alone and a rate of $10 \%$ in patients treated with curettage + bone graft or bone cement ${ }^{11}$. We excised the lesion and send for biopsy and histological finding were characteristic for fibroma was reported. Treatment options for fibroma include en bloc resection, simple curettage, and curettage with bone grafting or bone cement- polymethylmethacrylate placement.

CONCLUSION: Metacarpal fibroma is a rare bony tumor clinically and radiologically, it may be confused with other benign bone tumors, and for this reason the histopathological diagnosis is important. Tumor has high risk of recurrence. Treating fibroma with simple curettage offers the highest risk of recurrence, while en bloc resection has an almost negligible recurrence rate, but is associated with functional loss 2 . Some studies advocate curettage combined with autologous bone graft and report a low recurrence rate for these procedures 9 , 11. In our case we treated ossifying fibroma with wide resection ${ }^{12}$. Still we feel long term followup- atleast of 5 years is required to comment about recurrence.

\section{REFERENCES:}

1. Dahlin D. C. Bone tumors, Third edition, Charles C. Thomas, Springfield, Illinois, 1978, pp. 57-59

2. Huvos A. G. Tumors, diagnosis, treatment and prognosis, W. B. Saunders Co, Philadelphia , 1979, pp. 190-198

3. Jaffe H. L. Tumors and tumorous conditions of bones and joints Lea \& Febiger, Philadelphia, 1958, pp. 203-212

4. Lichtenstein L Bone tumors. Fifth edition, C. V. Mosby , St. Louis , 1977. pp. 63-77

5. Schajowicz F, Gallardo H , Chondromyxoid fibroma (fibromyxoid chondroma) of bone . J. Bone Joint Surg. , 1971, 53b , 198-216

6. Spjnt H. L., Dorfman H. D., Fechner R. E. Ackerman L. V. Tumors of bone and cartilage. In Atlas of tumor pathology, second series, fascicle 5. Washington D. C. Armed Forces Institute of Pathology, 1971, 50-59

7. Rahimi A, Beabout JW, Ivins JC, Dahlin DC, cancer, 30(1972) 726.

8. Schajowicz F, Gallardo H, J bone joint surg br, 53 (1971) 198.

9. Gherlinzoni F, Rock M, Picci P, J bone joint surg am, 65 (1983) 198.

10. Zillmer DA, Dorfman HD, Hum pathol, 20 (1989) 952.

11. Lersudi A, Mankin HJ, Mourikis , Hornicek FJ, clin orthop relat res, 12.005) 171

12. Bontemps E, Tillmann K, Handchir Mikrochir plast chir, 20 (1988) 321. 